\title{
Haemostatic and thrombo-embolic complications in pregnant women with COVID-19: a systematic review and critical analysis
}

Juliette Servante ${ }^{1 *}$ (D, Gill Swallow², Jim G. Thornton ${ }^{3}$, Bethan Myers ${ }^{4}$, Sandhya Munireddy ${ }^{4}$, A. Kinga Malinowski $i^{5}$, Maha Othman ${ }^{6,7}$, Wentao Li $i^{8}$, Keelin O'Donoghue ${ }^{9}$ and Kate F. Walker ${ }^{3}$

\begin{abstract}
Background: As pregnancy is a physiological prothrombotic state, pregnant women may be at increased risk of developing coagulopathic and/or thromboembolic complications associated with COVID-19.

Methods: Two biomedical databases were searched between September 2019 and June 2020 for case reports and series of pregnant women with a diagnosis of COVID-19 based either on a positive swab or high clinical suspicion where no swab had been performed. Additional registry cases known to the authors were included. Steps were taken to minimise duplicate patients. Information on coagulopathy based on abnormal coagulation test results or clinical evidence of disseminated intravascular coagulation (DIC), and on arterial or venous thrombosis, were extracted using a standard form. If available, detailed laboratory results and information on maternal outcomes were analysed.
\end{abstract}

Results: One thousand sixty-three women met the inclusion criteria, of which three $(0.28,95 \% \mathrm{Cl} 0.0$ to 0.6$)$ had arterial and/or venous thrombosis, seven $(0.66,95 \% \mathrm{Cl} 0.17$ to 1.1$)$ had $\mathrm{DIC}$, and a further three $(0.28,95 \% \mathrm{Cl} 0.0$ to 0.6) had coagulopathy without meeting the definition of DIC. Five hundred and thirty-seven women (56\%) had been reported as having given birth and $426(40 \%)$ as having an ongoing pregnancy. There were $17(1.6,95 \% \mathrm{Cl}$ 0.85 to 2.3 ) maternal deaths in which DIC was reported as a factor in two.

Conclusions: Our data suggests that coagulopathy and thromboembolism are both increased in pregnancies affected by COVID-19. Detection of the former may be useful in the identification of women at risk of deterioration.

Keywords: COVID-19, SARS-CoV-2, Pregnancy, Birth, Venous thrombosis, Arterial thrombosis, Coagulopathy, Disseminated intravascular coagulopathy, Haematological complications

\footnotetext{
* Correspondence: Juliette.servante@nottingham.ac.uk

'Department of Obstetrics and Gynaecology, Nottingham University Hospitals NHS Trust, Nottingham, UK

Full list of author information is available at the end of the article
}

C C The Author(s). 2021 Open Access This article is licensed under a Creative Commons Attribution 4.0 International License, which permits use, sharing, adaptation, distribution and reproduction in any medium or format, as long as you give appropriate credit to the original author(s) and the source, provide a link to the Creative Commons licence, and indicate if changes were made. The images or other third party material in this article are included in the article's Creative Commons licence, unless indicated otherwise in a credit line to the material. If material is not included in the article's Creative Commons licence and your intended use is not permitted by statutory regulation or exceeds the permitted use, you will need to obtain permission directly from the copyright holder. To view a copy of this licence, visit http://creativecommons.org/licenses/by/4.0/. The Creative Commons Public Domain Dedication waiver (http://creativecommons.org/publicdomain/zero/1.0/) applies to the data made available in this article, unless otherwise stated in a credit line to the data. 


\section{Background}

Outside pregnancy severe COVID-19 is prothrombotic and proinflammatory, and the presence of coagulopathy is associated with a poorer prognosis; $71 \%$ of patients who die have disseminated intravascular coagulopathy (DIC) as defined by the International Society on Thrombosis and Haemostasis (ISTH) criteria compared with $0.6 \%$ among survivors [1].

In the non-pregnant population, severe COVID-19 coagulopathy is characterised by a significantly elevated D-dimer concentration. Elevated D-dimers/fibrin degradation products are also seen in DIC as diagnosed according to the ISTH criteria [2, 3] and the pregnancy-specific DIC scoring system which has been developed to account for the relevant physiological adaptations [4]. However, unlike coagulopathy associated with other underlying causes, COVID-19 is less commonly associated with prolongation of prothrombin time (PT) and activate partial thromboplastin time (APTT) or thrombocytopenia [5, 6]. Fibrinogen appears to be at least initially well preserved although there have been reports of low fibrinogen, particularly in non-survivors $[1,7,8]$.

Accumulating data demonstrate increased risk of thromboembolism in COVID-19, predominantly in the most severe intensive care unit (ICU) cases [9-12]. Middledorp et al. found a $25 \%$ incidence at 7 days, rising to $48 \%$ at 14 days in ICU patients [9]. Similarly, Cui et al. demonstrated that 20/81 (25\%) of patients admitted to ICU developed thromboembolic complications, of which 8 died [10].

As pregnancy is already a physiologically hypercoagulable state, it seems likely that affected pregnant women would be at especially high risk of these complications. Current advice from the RCOG recommends that all pregnant women admitted with confirmed or suspected COVID-19 receive prophylactic low molecular weight heparin (LMWH), unless birth is expected within $12 \mathrm{~h}$, and continue this for 10 days following discharge [13]. Risk factors for thromboembolic complications in pregnancy are well documented.

Although the number of pregnant women with COVID-19 included in scientific reports as of 6th July 2020 stands at 6742 [14], many of these reports include the same or overlapping cases [15]. Potential duplicate publication is particularly challenging for reports from Wuhan, China; a city of 12 million people with 50 hospitals, 19 of which have reported cases of COVID-19 in pregnancy, and many of which have multiple names in translation [16]. In the West, hospitals and registries similarly often cite the same cases. Here, we have removed potentially duplicate reports in a conservative manner: when in doubt data were excluded.

In this systematic review, we aimed to determine two estimates:
1. The rate of arterial or venous thrombosis in pregnant women with confirmed or suspected COVID-19

2. The rate of acquired coagulopathy in pregnant women with confirmed or suspected COVID-19

\section{Methods}

Case reports and series of confirmed or suspected maternal COVID-19 in pregnancy were identified according to the methodology used by Walker et al. [17].

\section{Criteria for potentially eligible studies}

Studies were eligible for inclusion if they were case reports or case series, of pregnant women with confirmed COVID-19 infection and where the outcome of the pregnancy (either ongoing or delivered) was reported. There was no language restriction. We only included cases where either the mother had confirmed COVID19 based on a positive swab, or a high clinical suspicion of COVID-19 where a swab had not been taken e.g. symptoms and radiographic evidence in an area of high COVID-19 prevalence.

\section{Search strategy}

We identified all scientific case reports and case series of confirmed or suspected maternal COVID-19 in pregnancy. The basis of the list was a curated list kept by the senior author (JGT) on his personal blog since March 22nd. This is a curated list of primary sources based on a daily PubMed search supplemented by alerts from colleagues on social media. After April 8th this list was supplemented by formal daily searches by $\mathrm{KO}$ and KFW.

The search was undertaken between 8th April to May 2020 through the following electronic bibliographic databases (Medline, Embase and Maternity and Infant Care Database) and citation tracking on relevant studies. The search terms associated with COVID-19 used in bibliographic databases were adapted in databasespecific filters. The searches were re-run just before the final analyses and further studies retrieved for inclusion. The date of the last search was 05/06/2020. The search strategy is shown in Appendix 1. The dataset is available at: https://ripe-tomato.org/2020/05/15/covid-19-inpregnancy-101-onwards/.

\section{Selection of studies}

Titles and abstracts identified by the search strategy were assessed for inclusion by two reviewers (KW, KO). If there was disagreement about whether a report should be included, full text was obtained for that report.

For all potentially eligible studies full text copies were sought, and independently assessed for inclusion by two reviewers (KW, KO). Disagreements were resolved by discussion, and if agreement could not be 
reached the study was independently assessed by a third reviewer (JGT).

\section{Data extraction and data entry}

Data on study quality and content were extracted onto an Excel spread sheet, and checked (KW, JGT). Where data was missing, the first author of the paper was contacted by email $(n=4)$. Data was collected on maternal outcomes.

\section{Data analysis}

One-hundred-sixty-five papers were identified according to this methodology and 69 papers met inclusion criteria (see Fig. 1). Additional cases known to the authors were added from registries including the UK Obstetric Surveillance System (UKOSS) database, the East Midlands Research group (a group recently formed for the investigation of non-malignant haematological changes in pregnancy) and from the International Society on Thrombosis and Haemostasis' Pregnancy and COVID-19-Associated Coagulopathy (COV-PREG-COAG) Registry.

Coagulopathy events were recorded as stated by the authors. If haematological results were given, the DIC in pregnancy score was calculated, based on the prothrombin time, platelet count and fibrinogen levels. This scoring system has shown a sensitivity of $88 \%$ and a specificity of $96 \%$ for the diagnosis of DIC in pregnancy [4].
Few papers specifically stated negative findings for coagulopathy or thrombosis. Cases were therefore considered negative for these events if it was specified that there were no complications during the observed clinical course, or if patients were stated to have recovered/be recovering, or discharged without mention of coagulopathy or thrombosis.

Characteristics of each study were described and tabulated. Confidence intervals for the outcomes given were calculated using software available at: https://epitools. ausvet.com.au/ciproportion.

\section{Results}

Details for 1063 women with COVID-19 in pregnancy have been reported, where maternal outcomes were provided. Of these, three $(0.28,95 \%$ CI 0.0 to 0.6$))$ have had thromboembolic disease, seven $(0.66,95 \%$ CI 0.17 to 1.1$)$ have been diagnosed with DIC, with another three $(0.28$, 95\% CI 0.0 to 0.6$)$ ) noted to have a coagulopathy. Five hundred and thirty-seven (56\%) have been reported as recovered/recovering and having given birth and 426 (40\%) have been reported as recovered/recovering with ongoing pregnancy (Table 1). In addition, Pereira et al described 2/60 patients with deep vein thrombosis (DVT); however, this report was discounted from the above totals (and Table 1) due to lack of reported pregnancy outcomes [7].

Tables 2 and 3 provide summaries of reported cases of thrombosis and coagulopathy respectively, in pregnant

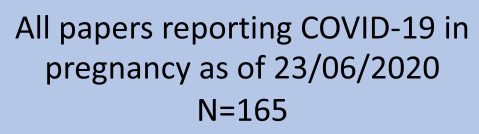

Exclusions applied

- Papers with confirmed or presumed overlap in case reporting $(\mathrm{N}=50)$

- Papers with nil confirmed cases* of COVID-19 in pregnancy $(\mathrm{N}=5)$

- Papers with nil credible source (i.e nil trace of origin of data) $(\mathrm{N}=1)$

- Papers with nil case-specific details of maternal/pregnancy outcome $(\mathrm{N}=40)$

\section{Papers included in analysis} $\mathrm{N}=69$

\section{*Confirmed COVID-19 based on a positive swab or high clinical suspicion of COVID-} 19 where a swab had not been taken e.g. symptoms and radiographic evidence.

Fig. 1 Flow chart of papers included in analysis. Papers were identified between 08/02/20 and 05/06/20 using methodology described by Walker et al. The original dataset is available at https://ripe-tomato.org/2020/05/15/covid-19-in-pregnancy-101-onwards/. Exclusion criteria were applied, and 69 papers were included in the final analysis 
Table 1 Summary of all reported cases with haemostatic complications, coagulopathies or DIC in pregnant women with confirmed COVID-19 infection

\begin{tabular}{|c|c|c|c|c|c|c|c|c|}
\hline Location & $\begin{array}{l}\text { Source } \\
\text { (Study } \\
\text { number } \\
\text { as per } \\
\text { ripe-tomato.org } \\
\text { or database) }\end{array}$ & $\begin{array}{l}\text { Pregnant } \\
\text { women with } \\
\text { confirmed } \\
\text { COVID-19 } \\
\text { infection with } \\
\text { outcomes } \\
\text { reported }\end{array}$ & $\begin{array}{l}\text { Women } \\
\text { who } \\
\text { required } \\
\text { critical } \\
\text { care. } \\
\text { N/A not } \\
\text { available }\end{array}$ & $\begin{array}{l}\text { Women } \\
\text { who } \\
\text { delivered, } \\
\text { presumed } \\
\text { healthy }^{1}\end{array}$ & $\begin{array}{l}\text { Women with } \\
\text { ongoing } \\
\text { pregnancies, } \\
\text { presumed } \\
\text { healthy }^{1}\end{array}$ & $\begin{array}{l}\text { Venous } \\
\text { thrombotic } \\
\text { events }\end{array}$ & $\begin{array}{l}\text { Arterial } \\
\text { thrombotic } \\
\text { events }\end{array}$ & $\begin{array}{l}\text { Disseminated } \\
\text { intravascular } \\
\text { coagulation } \\
\text { (DIC) events }\end{array}$ \\
\hline \multicolumn{9}{|l|}{ China } \\
\hline $\begin{array}{l}\text { Zhongnan Hospital of } \\
\text { Wuhan University }\end{array}$ & 1 & 9 & 0 & 9 & 0 & 0 & 0 & 0 \\
\hline $\begin{array}{l}\text { Union Hospital, Tongji } \\
\text { Medical College, } \\
\text { Huazhong University of } \\
\text { Science and Technology }\end{array}$ & $2 a$ & 3 & 0 & 3 & 0 & 0 & 0 & 0 \\
\hline $\begin{array}{l}\text { The first Affiliated Hospital, } \\
\text { College of Medicine, } \\
\text { Zhejiang University }\end{array}$ & 4 & 1 & 0 & 1 & 0 & 0 & 0 & 0 \\
\hline $\begin{array}{l}\text { Union Hospital, Tongji } \\
\text { Medical College, } \\
\text { Huazhong University of } \\
\text { Science and Technology }\end{array}$ & 6 & 15 & 0 & 11 & 4 & 0 & 0 & 0 \\
\hline $\begin{array}{l}\text { Qingdao Women and } \\
\text { Children's Hospital, } \\
\text { Qingdao }\end{array}$ & 7 & 1 & 0 & 0 & 1 & 0 & 0 & 0 \\
\hline $\begin{array}{l}\text { Tongji Hospital, Tongji } \\
\text { Medical College, } \\
\text { Huazhong University of } \\
\text { Science and Technology }\end{array}$ & 15 & 7 & 0 & 7 & 0 & 0 & 0 & 0 \\
\hline $\begin{array}{l}\text { Affiliated Infectious } \\
\text { Hospital of Soochow } \\
\text { University, Suzhou }\end{array}$ & 19 & 1 & 1 & 1 & 0 & 0 & 0 & 0 \\
\hline $\begin{array}{l}\text { Maternal and Child } \\
\text { Hospital of Hubei Province }\end{array}$ & 30 & 34 & 0 & 34 & 0 & 0 & 0 & 0 \\
\hline $\begin{array}{l}\text { Beijing YouAn Hospital, } \\
\text { Capital Medical University }\end{array}$ & 34 & 1 & 0 & 1 & 0 & 0 & 0 & 0 \\
\hline $\begin{array}{l}\text { Renmin Hospital of Wuhan } \\
\text { University }\end{array}$ & 36 & $17^{3 a}$ & N/A & 14 & 0 & 0 & 0 & 0 \\
\hline $\begin{array}{l}\text { Renmin Hospital of Wuhan } \\
\text { University }\end{array}$ & 37 & 3 & 0 & 3 & 0 & 0 & 0 & 0 \\
\hline $\begin{array}{l}\text { No } 2 \text { People's Hospital of } \\
\text { Hefei City Affiliated to } \\
\text { Anhui Medical University }\end{array}$ & 62 & 1 & 0 & 1 & 0 & 0 & 0 & 0 \\
\hline Central Hospital of Wuhan & 73 & $28^{4 e}$ & N/A & 22 & 2 & 0 & 0 & 0 \\
\hline $\begin{array}{l}\text { Xiaolan People's Hospital } \\
\text { of Zhongshan, Guandong }\end{array}$ & 81 & 1 & 1 & 1 & 0 & 0 & 0 & 1 \\
\hline \multicolumn{9}{|l|}{ USA } \\
\hline $\begin{array}{l}\text { MedStar Washington } \\
\text { Hospital Center, DC }\end{array}$ & 21 & 1 & 0 & 1 & 0 & 0 & 0 & 0 \\
\hline $\begin{array}{l}\text { Newark Beth Israel Medical } \\
\text { Centre, New Jersey }\end{array}$ & 28 & $2^{a, b}$ & N/A & 0 & 0 & 0 & 0 & 0 \\
\hline $\begin{array}{l}\text { Saint Barnabas Medical } \\
\text { Center, Livingston, New } \\
\text { Jersey }\end{array}$ & 111 & 1 & 1 & 1 & 0 & 0 & 0 & 0 \\
\hline $\begin{array}{l}\text { Morristown medical } \\
\text { centre, St Peter's University } \\
\text { Medical Centre, New } \\
\text { Jersey }\end{array}$ & 159 & 3 & 3 & 0 & 3 & $0^{*}$ & 0 & 0 \\
\hline
\end{tabular}


Table 1 Summary of all reported cases with haemostatic complications, coagulopathies or DIC in pregnant women with confirmed COVID-19 infection (Continued)

\begin{tabular}{|c|c|c|c|c|c|c|c|c|}
\hline Location & $\begin{array}{l}\text { Source } \\
\text { (Study } \\
\text { number } \\
\text { as per } \\
\text { ripe-tomato.org } \\
\text { or database) }\end{array}$ & $\begin{array}{l}\text { Pregnant } \\
\text { women with } \\
\text { confirmed } \\
\text { COVID-19 } \\
\text { infection with } \\
\text { outcomes } \\
\text { reported }\end{array}$ & $\begin{array}{l}\text { Women } \\
\text { who } \\
\text { required } \\
\text { critical } \\
\text { care. } \\
\text { N/A not } \\
\text { available }\end{array}$ & $\begin{array}{l}\text { Women } \\
\text { who } \\
\text { delivered, } \\
\text { presumed } \\
\text { healthy }^{1}\end{array}$ & $\begin{array}{l}\text { Women with } \\
\text { ongoing } \\
\text { pregnancies, } \\
\text { presumed } \\
\text { healthy }^{1}\end{array}$ & $\begin{array}{l}\text { Venous } \\
\text { thrombotic } \\
\text { events }\end{array}$ & $\begin{array}{l}\text { Arterial } \\
\text { thrombotic } \\
\text { events }\end{array}$ & $\begin{array}{l}\text { Disseminated } \\
\text { intravascular } \\
\text { coagulation } \\
\text { (DIC) events }\end{array}$ \\
\hline $\begin{array}{l}\text { "Network's } 2 \text { largest } \\
\text { hospitals" in New Jersey: } \\
\text { Likely Hackensack } \\
\text { University Medical Centre, } \\
\text { Rutgers New Jersey } \\
\text { Medical School Newark, } \\
\text { Seton Hall University } \\
\text { Nutley, Jersey Shore } \\
\text { University Medical Centre, } \\
\text { Neptune, }\end{array}$ & 149 & 8 & 8 & 7 & 1 & 0 & 0 & 0 \\
\hline $\begin{array}{l}\text { Good Samaritan Hospital, } \\
\text { Cincinnati, Ohio }\end{array}$ & 50 & $1^{b}$ & 1 & 0 & 0 & 0 & 0 & $0^{* *}$ \\
\hline $\begin{array}{l}\text { Lexington Medical Centre, } \\
\text { West Columbia, South } \\
\text { Carolina }\end{array}$ & 60 & 1 & 0 & 0 & 1 & 0 & 0 & 0 \\
\hline $\begin{array}{l}\text { Hospital of the University } \\
\text { of Pennsylvania }\end{array}$ & 65 & $5^{b}$ & 5 & 3 & 1 & 1 & 0 & 0 \\
\hline $\begin{array}{l}\text { Washington University in } \\
\text { St Louis, Missouri }\end{array}$ & 69 & 1 & 1 & 1 & 0 & 0 & 0 & 0 \\
\hline $\begin{array}{l}\text { Beaumont Hospital } \\
\text { Dearborn, Dearborn, } \\
\text { Michigan }\end{array}$ & 123 & 16 & 0 & 10 & 6 & 0 & 0 & 0 \\
\hline $\begin{array}{l}\text { Henry Ford Hospital } \\
\text { Department of Obstetrics } \\
\text { and Gynecology, Detroit, } \\
\text { Michigan (distinct case } \\
\text { from 123) }\end{array}$ & 87 & 1 & 1 & 0 & 1 & 0 & $0^{*}$ & 0 \\
\hline $\begin{array}{l}\text { University of California, San } \\
\text { Francisco, California }\end{array}$ & 89 & 1 & 1 & 1 & 0 & 0 & 0 & 0 \\
\hline $\begin{array}{l}\text { Stanford University } \\
\text { Hospital, California }\end{array}$ & 115 & 1 & 0 & 1 & 0 & 0 & 0 & 0 \\
\hline $\begin{array}{l}\text { New York University, } \\
\text { Winthrop hospital, } \\
\text { Langone health }\end{array}$ & 91 & 1 & 1 & 1 & 0 & 0 & 0 & 0 \\
\hline $\begin{array}{l}\text { New York University, } \\
\text { Langone Health (distinct } \\
\text { case) }\end{array}$ & 98 & $1^{c}$ & 0 & 0 & 0 & 0 & 0 & 0 \\
\hline $\begin{array}{l}\text { Weil Cornell Medicine, } \\
\text { New York** }\end{array}$ & 99 & $20^{h}$ & 0 & 19 & 0 & 0 & 0 & 0 \\
\hline Norwell Group, New York & $\begin{array}{l}118 \\
162\end{array}$ & $13^{2 g, a}$ & 13 & 5 & 5 & 0 & 0 & $0^{* *}$ \\
\hline $\begin{array}{l}\text { New York University, } \\
\text { Langone health }\end{array}$ & 152 & 2 & 2 & 2 & 0 & 0 & 0 & 0 \\
\hline $\begin{array}{l}\text { Six hospital systems in } \\
\text { Washington state }\end{array}$ & 102 & 46 & 1 & 8 & 38 & 0 & 0 & 0 \\
\hline $\begin{array}{l}\text { University of Tennessee } \\
\text { Health Science Center, } \\
\text { Nashville, United States }\end{array}$ & 112 & 1 & 1 & 0 & 1 & 0 & 0 & 0 \\
\hline Yale School of Medicine & 155 & $1^{e}$ & N/A & 0 & 0 & 0 & 0 & 1 \\
\hline $\begin{array}{l}\text { Advocate Good Samaritan } \\
\text { Hospital, Illinois, United } \\
\text { States^}\end{array}$ & COV-PREG-COAG & 1 & 0 & 1 & 0 & 0 & 0 & 0 \\
\hline
\end{tabular}


Table 1 Summary of all reported cases with haemostatic complications, coagulopathies or DIC in pregnant women with confirmed COVID-19 infection (Continued)

\begin{tabular}{|c|c|c|c|c|c|c|c|c|}
\hline Location & $\begin{array}{l}\text { Source } \\
\text { (Study } \\
\text { number } \\
\text { as per } \\
\text { ripe-tomato.org } \\
\text { or database) }\end{array}$ & $\begin{array}{l}\text { Pregnant } \\
\text { women with } \\
\text { confirmed } \\
\text { COVID-19 } \\
\text { infection with } \\
\text { outcomes } \\
\text { reported }\end{array}$ & $\begin{array}{l}\text { Women } \\
\text { who } \\
\text { required } \\
\text { critical } \\
\text { care. } \\
\text { N/A not } \\
\text { available }\end{array}$ & $\begin{array}{l}\text { Women } \\
\text { who } \\
\text { delivered, } \\
\text { presumed } \\
\text { healthy }^{1}\end{array}$ & $\begin{array}{l}\text { Women with } \\
\text { ongoing } \\
\text { pregnancies, } \\
\text { presumed } \\
\text { healthy }^{1}\end{array}$ & $\begin{array}{l}\text { Venous } \\
\text { thrombotic } \\
\text { events }\end{array}$ & $\begin{array}{l}\text { Arterial } \\
\text { thrombotic } \\
\text { events }\end{array}$ & $\begin{array}{l}\text { Disseminated } \\
\text { intravascular } \\
\text { coagulation } \\
\text { (DIC) events }\end{array}$ \\
\hline St Joseph Hospital, Denver & 156 & 1 & 0 & 1 & 0 & 0 & 0 & 0 \\
\hline \multicolumn{9}{|l|}{ Canada } \\
\hline $\begin{array}{l}\text { Mount Sinai Hospital, } \\
\text { Toronto }\end{array}$ & 48 & 1 & 0 & 1 & 0 & 0 & 0 & 1 \\
\hline \multicolumn{9}{|l|}{ Honduras } \\
\hline $\begin{array}{l}\text { Hospital Escuela de } \\
\text { Tegucigalpa }\end{array}$ & 18 & 1 & 0 & 1 & 0 & 0 & 0 & 0 \\
\hline \multicolumn{9}{|l|}{ Sweden } \\
\hline $\begin{array}{l}\text { Southern General Hospital, } \\
\text { Stockholm }\end{array}$ & 20 & 1 & 0 & 1 & 0 & 0 & 0 & 0 \\
\hline \multicolumn{9}{|l|}{ France } \\
\hline $\begin{array}{l}\text { Antoine Beclere Hospital, } \\
\text { Clamart }\end{array}$ & 48 & 1 & N/A & 1 & 0 & 0 & 0 & 1 \\
\hline $\begin{array}{l}\text { Hospitaux Universitaires de } \\
\text { Strasbourg }\end{array}$ & 161 & $54^{\mathrm{a}, \mathrm{b}, \mathrm{d}}$ & 5 & 20 & 31 & 0 & 0 & 0 \\
\hline \multicolumn{9}{|l|}{ Canary Islands } \\
\hline $\begin{array}{l}\text { Hospitalario Universitario } \\
\text { Insular Materno Infantil, } \\
\text { Gran Canaria }\end{array}$ & 53 & 1 & 1 & 1 & 0 & 0 & 0 & 0 \\
\hline \multicolumn{9}{|l|}{ Italy } \\
\hline $\begin{array}{l}\text { Fondazione Policlinico } \\
\text { Universitario A. Gemelli } \\
\text { IRCCS, Rome, Italy }\end{array}$ & 76 & $7^{c}$ & N/A & 4 & 2 & 0 & 0 & 0 \\
\hline $\begin{array}{l}12 \text { Italian hospitals (non- } \\
\text { overlapping with others in } \\
\text { table) }\end{array}$ & 117 & 26 & 14 & 6 & 20 & 0 & 0 & 0 \\
\hline Parma Hospital, Italy & 109 & 4 & $\mathrm{~N} / \mathrm{A}$ & 4 & 0 & 0 & 0 & 0 \\
\hline $\begin{array}{l}6 \text { hospitals of Azienda USL } \\
62 \text { "Toscana Nord Ovest" } \\
\text { [ATNO] (Tuscany), and Gaslini } \\
\text { Children's Hospital (Genoa, } \\
\text { Liguria) }\end{array}$ & 133 & 3 & 0 & 3 & 0 & 0 & 0 & 0 \\
\hline \multicolumn{9}{|l|}{ UK } \\
\hline Portland Hospital London & 82 & 8 & 0 & 8 & 0 & 0 & 0 & 0 \\
\hline $\begin{array}{l}\text { East Midlands Research } \\
\text { group (University Hospitals } \\
\text { of Leicester and } \\
\text { Nottingham University } \\
\text { Hospitals) }\end{array}$ & $\begin{array}{l}\text { East Midlands } \\
\text { Research Group }\end{array}$ & $30^{\mathrm{b}, \mathrm{g}}$ & 2 & 21 & 7 & 0 & 0 & 1 \\
\hline $\begin{array}{l}\text { UK (Nationwide)- UKOSS } \\
\text { database with case } \\
\text { information as per paper } \\
107 .\end{array}$ & UKOSS + 107 & $427^{5 g}$ & 41 & 261 & 161 & 1 & 1 & 0 \\
\hline \multicolumn{9}{|l|}{ Belgium } \\
\hline $\begin{array}{l}\text { Cliniques Universitaires, St } \\
\text { Luc, Brussels, }\end{array}$ & 100 & 1 & 0 & 1 & 0 & 0 & 0 & 0 \\
\hline $\begin{array}{l}4 \text { Obstetric units in North } \\
\text { East Flanders }\end{array}$ & 128 & 13 & 0 & 13 & 0 & 0 & 0 & 0 \\
\hline
\end{tabular}


Table 1 Summary of all reported cases with haemostatic complications, coagulopathies or DIC in pregnant women with confirmed COVID-19 infection (Continued)

\begin{tabular}{|c|c|c|c|c|c|c|c|c|}
\hline Location & $\begin{array}{l}\text { Source } \\
\text { (Study } \\
\text { number } \\
\text { as per } \\
\text { ripe-tomato.org } \\
\text { or database) }\end{array}$ & $\begin{array}{l}\text { Pregnant } \\
\text { women with } \\
\text { confirmed } \\
\text { COVID-19 } \\
\text { infection with } \\
\text { outcomes } \\
\text { reported }\end{array}$ & $\begin{array}{l}\text { Women } \\
\text { who } \\
\text { required } \\
\text { critical } \\
\text { care. } \\
\text { N/A not } \\
\text { available }\end{array}$ & $\begin{array}{l}\text { Women } \\
\text { who } \\
\text { delivered, } \\
\text { presumed } \\
\text { healthy }^{1}\end{array}$ & $\begin{array}{l}\text { Women with } \\
\text { ongoing } \\
\text { pregnancies, } \\
\text { presumed } \\
\text { healthy }^{1}\end{array}$ & $\begin{array}{l}\text { Venous } \\
\text { thrombotic } \\
\text { events }\end{array}$ & $\begin{array}{l}\text { Arterial } \\
\text { thrombotic } \\
\text { events }\end{array}$ & $\begin{array}{l}\text { Disseminated } \\
\text { intravascular } \\
\text { coagulation } \\
\text { (DIC) events }\end{array}$ \\
\hline
\end{tabular}

Portugal

Hospital Pedro Hispano 105

Porto (distinct case) 94

Portugal (distinct case) 74

Netherlands

Netherlands COVID-19 14

registry

Germany

$$
\text { Ulm university }
$$

Spain

Jaen

Barcelona

South Korea

Daegu Fatimal Hospital

Japan

Keio University Hospital, $\quad 144$

Tokyo

Turkey

Ankara University Faculty

of Medicine,

Sehit Prof Dr. Ilhan Varank 146

Sancaktepe Training and

Research Hospital, Istanbul

Necmettin Erkbakan $\quad 145$

University, Konya

127

158

140

Jordan

Jordan

Australia

Gold Coast University $\quad 45$ Hospital

India

Designated Covid Hospital 58 Iran

Tehran/Rasht/Qom/Zanjan

Imam Khomeini Hospital,

Sari, Iran

Imam Reza Hospital of 101

Tabriz, Iran

Thailand

Thailand (reported by

ministry of public health)

Russia

12

1

1

$176^{\mathrm{d}, \mathrm{g}, \mathrm{i}}$

7

49

124

0

2

0

$$
0
$$

0

0

\section{4}

8

0

8

0

4

\section{0}

0

\section{0}

0

0

0 
Table 1 Summary of all reported cases with haemostatic complications, coagulopathies or DIC in pregnant women with confirmed COVID-19 infection (Continued)

\begin{tabular}{|c|c|c|c|c|c|c|c|c|}
\hline Location & $\begin{array}{l}\text { Source } \\
\text { (Study } \\
\text { number } \\
\text { as per } \\
\text { ripe-tomato.org } \\
\text { or database) }\end{array}$ & $\begin{array}{l}\text { Pregnant } \\
\text { women with } \\
\text { confirmed } \\
\text { COVID-19 } \\
\text { infection with } \\
\text { outcomes } \\
\text { reported }\end{array}$ & $\begin{array}{l}\text { Women } \\
\text { who } \\
\text { required } \\
\text { critical } \\
\text { care. } \\
\text { N/A not } \\
\text { available }\end{array}$ & $\begin{array}{l}\text { Women } \\
\text { who } \\
\text { delivered, } \\
\text { presumed } \\
\text { healthy }^{1}\end{array}$ & $\begin{array}{l}\text { Women with } \\
\text { ongoing } \\
\text { pregnancies, } \\
\text { presumed } \\
\text { healthy }^{1}\end{array}$ & $\begin{array}{l}\text { Venous } \\
\text { thrombotic } \\
\text { events }\end{array}$ & $\begin{array}{l}\text { Arterial } \\
\text { thrombotic } \\
\text { events }\end{array}$ & $\begin{array}{l}\text { Disseminated } \\
\text { intravascular } \\
\text { coagulation } \\
\text { (DIC) events }\end{array}$ \\
\hline \multicolumn{9}{|l|}{ Center^ } \\
\hline \multicolumn{9}{|l|}{ UAE } \\
\hline $\begin{array}{l}\text { Al Ain Hospital, United } \\
\text { Arab Emirates } \wedge\end{array}$ & COV-PREG-COAG & 1 & 0 & 1 & 0 & 0 & 0 & 0 \\
\hline Location & & $\begin{array}{l}\text { Pregnant } \\
\text { women with } \\
\text { confirmed } \\
\text { COVID-19 in- } \\
\text { fection with } \\
\text { outcomes } \\
\text { reported }\end{array}$ & $\begin{array}{l}\text { Women } \\
\text { who } \\
\text { required } \\
\text { critical } \\
\text { care. } \\
\text { N/A not } \\
\text { available }\end{array}$ & $\begin{array}{l}\text { Women } \\
\text { who } \\
\text { delivered, } \\
\text { presumed } \\
\text { healthy }^{1}\end{array}$ & $\begin{array}{l}\text { Women with } \\
\text { ongoing } \\
\text { pregnancies, } \\
\text { presumed } \\
\text { healthy }^{1}\end{array}$ & $\begin{array}{l}\text { Venous } \\
\text { thrombotic } \\
\text { events }\end{array}$ & $\begin{array}{l}\text { Arterial } \\
\text { thrombotic } \\
\text { events }\end{array}$ & $\begin{array}{l}\text { Disseminated } \\
\text { intravascular } \\
\text { coagulation } \\
\text { (DIC) events }\end{array}$ \\
\hline Total & & 1063 & $\begin{array}{l}132 / \\
1033\end{array}$ & 593 & 426 & 3 & 1 & 7 \\
\hline
\end{tabular}

a-remains inpatient (6), b-remains inpatient- stated to be on ITU/ventilator (8), c-Pregnancy loss before 24 weeks (3), d-pregnancy loss (gestation not stated) (2), etermination before 24 weeks (due to COVID-19) (5), f- termination before 24 weeks (other reason) (1), g-patient died (17), h-readmission with nil further details (1), i-molar pregnancy (1)

*line thrombosis noted (see Table 2)

**Additional coagulopathy noted (see Table 3)

*** Isolated abnormal coagulation parameters- not specified further

${ }^{1}$ Few papers specifically stated negative findings for coagulopathy or thrombosis. Cases were therefore considered negative for these events if it was stated that there were no complications during the observed clinical course, or if patients were stated to have recovered/be recovering, or discharged without mention of coagulopathy or thrombosis

women confirmed or highly-suspected to have COVID19 as taken from Table 1.

Of 1063 pregnant women included in our current study, there were 17 deaths $(1.6,95 \% \mathrm{CI} 0.85$ to 2.3$)$. DIC was reported in two of these cases (12\%). We also noted a higher incidence of thrombotic events in non-survivors, with pulmonary embolism occurring in two cases (distinct to the cases of DIC) and concurrent basilar artery thrombosis in one case. One hundred and thirty two/1033 (13.0\%) women with COVID-19 in this study required admission to ICU.

Platelet levels and D-dimers were reported in several cases where haematological results did not meet the criteria for DIC and patients had not been stated to have a coagulopathy. In addition to cases noted to have a coagulopathy, D-dimer was noted to be raised (as reported by authors or above $0.5 \mathrm{mg} / \mathrm{l}$ ) in 31 of 38 cases [18-33], and from the COV-PREG-COAG Registry] where a value was reported or commented on. Platelets were low (as reported by authors or $<100$ ) in 15 of 102 cases where a value was reported or commented on $[18,19$, $21,23,24,27-30,33-40]$, also cases from the COVPREG-COAG Registry] (see Appendix 2).

\section{Discussion}

\section{Statement of principle findings}

Haemostatic and thromboembolic complications have been reported in 0.98 and $0.28 \%$ of pregnant women with COVID-19 infection respectively. The absolute risk of thromboembolic complications in pregnant women without COVID-19 is $0.1 \%$ [41]. Estimates of the incidence of DIC in pregnant women range between 0.03 to $0.35 \%$ [42]. Our findings suggest that the risk of haemostatic and thromboembolic complications are higher in pregnant women with COVID-19 infection than in pregnant women without COVID-19 infection.

\section{Strengths and limitations}

Our review is the largest reported to date, even following removal of potential duplicates. The precision of our estimates is therefore greater.

Many primary studies were case reports or hospitalbased series, which are at risk of bias towards cases or findings of interest, resulting in potential overestimation of complications. On the other hand, few papers specifically stated that there were no haemostatic complications in each case. Our assumption that this means an absence of complications may result in an underestimate, as theoretically complications may have been present, but not reported.

The DIC score used to identify cases from laboratory findings is a composite of prothrombin time, platelet counts and fibrinogen levels [4]. However, coagulopathy in COVID-19 is associated with a modest change in these parameters [5], meaning that the DIC score alone 


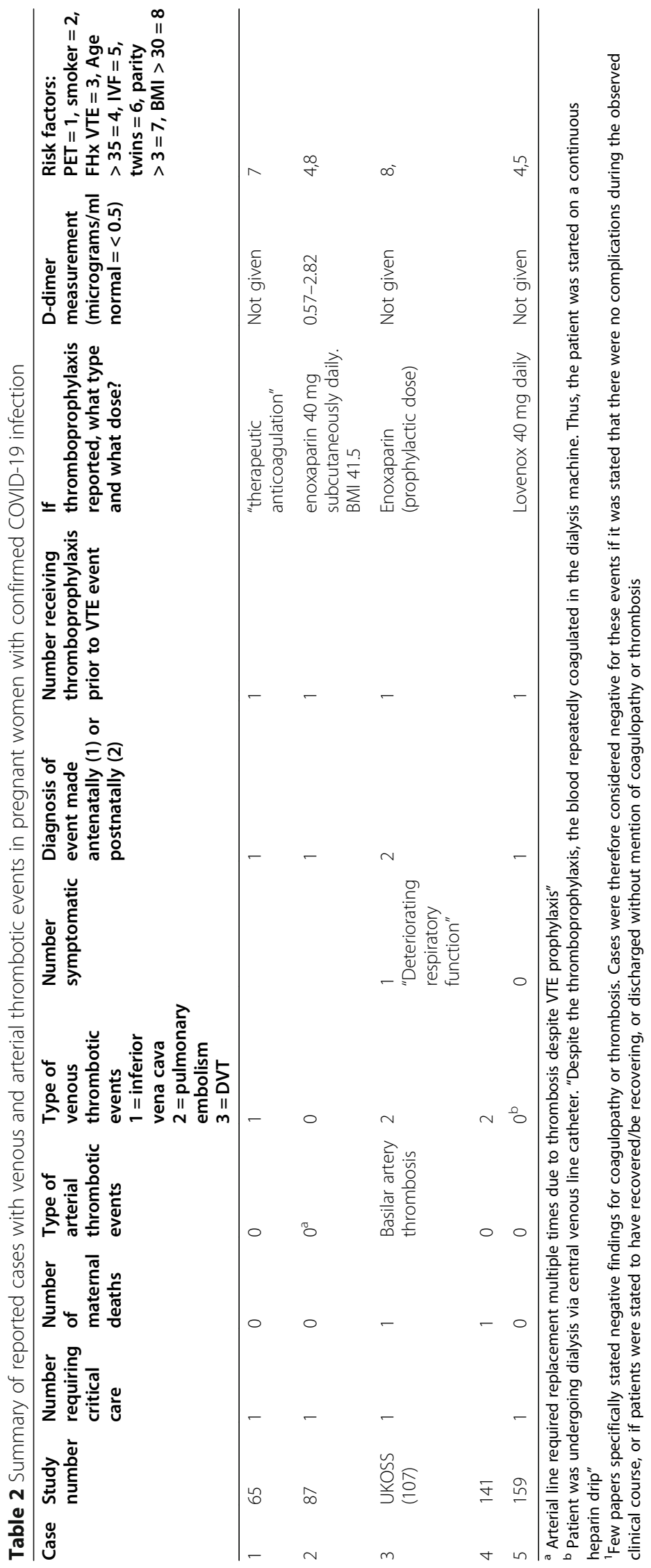




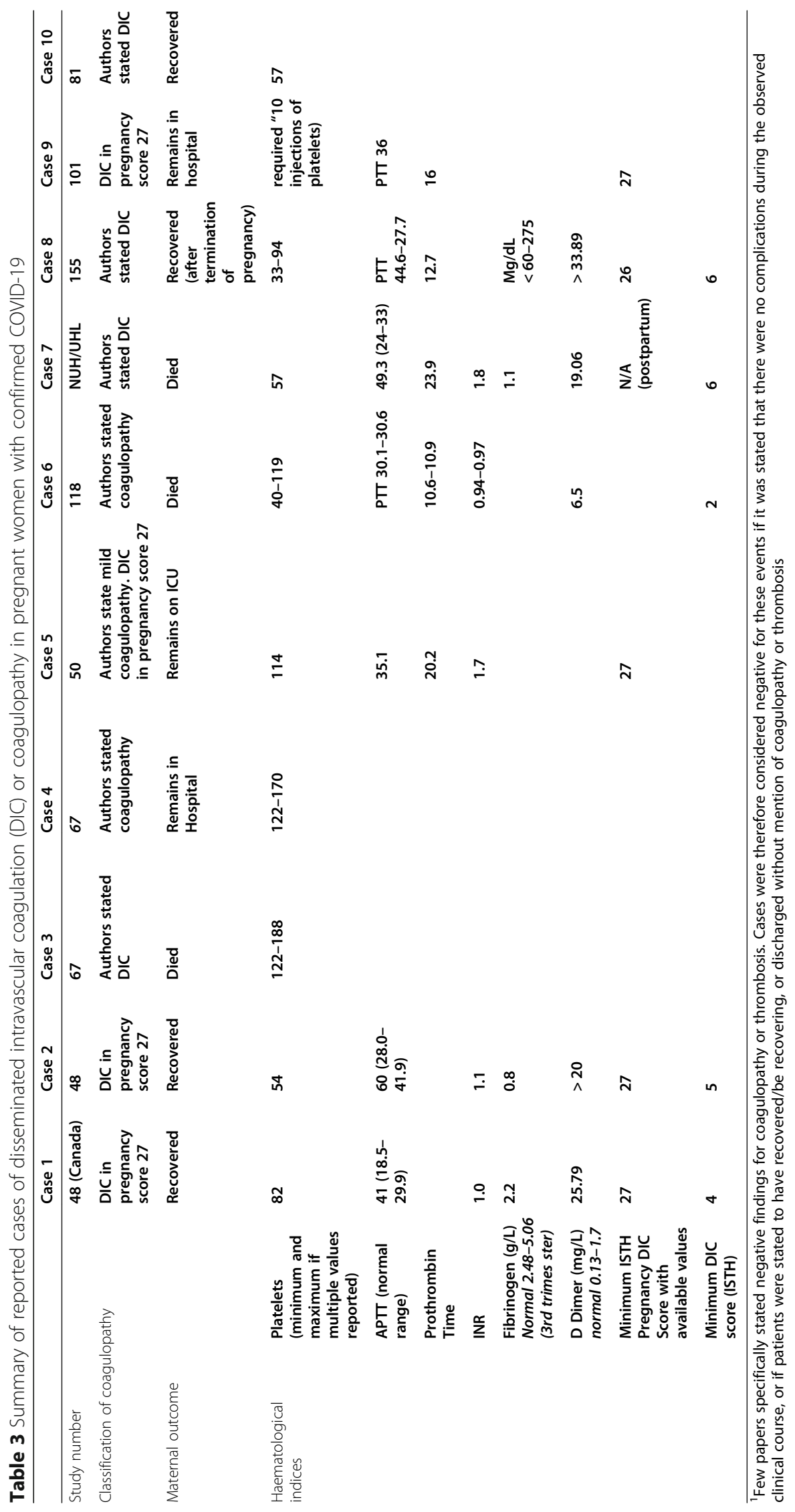


may be less accurate as a measure of COVID-19 coagulopathy in pregnancy. In addition, many authors did not report fibrinogen levels or prothrombin time, which will have falsely lowered our rate estimate of coagulopathy. D-dimer, like C-reactive protein (CRP), is an acute phase reactant, which can be elevated in trauma or any inflammatory condition. Elevated D-dimer levels are difficult to interpret, as the etiology of their rise can be multifactorial. D-dimer elevations can occur during an uncomplicated pregnancy, though typically they are not as pronounced as in some of the cases in this study, where the values were reported. Pneumonia as well has been associated with high D-dimer levels, as have thromboembolic events. As reported in Pereira et al, pregnant women who were classified as having severe clinical features of pneumonia in COVID-19 had higher D-dimer and CRP [7]. On the other hand, significant elevations of D-dimer were also noted in two reported cases of COVID-19 associated coagulopathy in pregnancy, neither of which were complicated by pneumonia or significant respiratory compromise [42]. While lack of standardisation of D-dimer thresholds in pregnancy renders interpretation challenging, in these two cases Ddimer levels were grossly elevated, at 17- and 12- fold the upper limit of normal [42].

The efficacy of D-dimer in the diagnosis of pulmonary embolism (PE) in pregnancy has been investigated, with conflicting results. The DiPEP (diagnosis of PE in pregnancy) group concluded, using D-dimer measurement by ELISA (counted as negative if $<400 \mathrm{ng} / \mathrm{ml}$ ) and using Innovance technology (reference range $1-1.3 \mathrm{mg} / \mathrm{L}$ ), that D-Dimer was not useful for the diagnosis of PE in the context of pregnancy [43]. However, Van der Pol et al. reported that D-dimer measurement could be used in order to rule out PE in this group [44], using a cut of value of $>1000 \mathrm{ng} / \mathrm{ml}$ if nil clinical criteria were met, or $<500 \mathrm{ng} / \mathrm{ml}$ where wither there were clinical signs of either deep vein thrombosis; haemoptysis or where PE was the most likely diagnosis. Thus, the potential prognostic value of $\mathrm{D}$-dimer in pregnancy in the setting of COVID-19 cannot be dismissed outright and deserves further investigation. Additionally, other tools for assessing hypercoagulability or other forms of coagulopathy such as Thromboelastography ${ }^{\text {Tm }} /$ /Thromboelastometry $^{\text {mat }}$ are worth evaluating. An ISTH review and recommendation for the use of these technologies in obstetrics has recently been published [45].

\section{Comparison with previous studies}

Sentilhes [33] found no cases of thromboembolic disease or thrombocytopenia among 54 pregnant women with COVID-19 including five women who were admitted to ICU in Strasbourg. Guan [46] reported one case of DIC among 1099 cases of laboratory confirmed COVID-19 in non-pregnant patients of all ages ( $0.1 \%$ of cases). Tang [1] noted a higher incidence of coagulopathy in nonsurvivors which is in keeping with our findings. Whilst uncommon in pregnant women with COVID-19, our data suggests that the identification of haemostatic and coagulopathic changes may have value in the identification of women at risk of deterioration.

\section{Conclusion}

\section{Implications for clinical practice}

Our findings suggest that haematological complications are more commonly observed in pregnant women with COVID-19 infection (1.26\%) than in pregnant women without $(0.45 \%)$ and support the current advice from the RCOG recommending that all pregnant women admitted with confirmed or suspected COVID-19 receive prophylactic low molecular weight heparin (LMWH), unless birth is expected within $12 \mathrm{~h}$, and continue this for 10 days following discharge.

Despite findings of elevated D-dimer in patients who have tested positive for COVID-19 outside of pregnancy, the occurrence of DIC and thrombotic events is infrequently reported [6]. We have found this to also be the case where COVID-19 is described in pregnancy; perhaps in part due the resultant coagulopathy being distinct from DIC and/or secondary to a lack of standardised cut off values for coagulation parameters for the diagnosis of coagulopathy in COVID-19 in the context of pregnancy. Nonetheless, identification of haemostatic and thrombotic complications may still be of clinical importance in recognizing pregnant patients who are at a higher risk of mortality from COVID-19.

To diagnose coagulopathy in a pregnant woman with COVID-19, we would recommend checking a full blood count, D dimer/fibrin degradation products (FDP), clotting screen and fibrinogen and using these parameters to calculate the pregnancy related DIC score. These parameters are useful if the woman needs delivery and can guide blood product support. Othman et al provide practical suggestions on interpretation of these laboratory parameters based on expert consensus [8].

Despite findings of DIC, there is no evidence that correcting abnormal coagulation parameters in patients who are not actively bleeding is beneficial. This advice covers all patients with COVID associated DIC. The only difference for pregnant women would be if they required delivery. Do not use tranexamic acid; recovery from DIC is dependent on endogenous fibrinolysis to break down the disseminated thrombi. This process is inhibited by tranexamic acid, an anti-fibrinolytic drug. If there is bleeding associated with DIC give blood product replacement.

Given the increased chances of thrombosis in a normal pregnancy there needs to be a high index of suspicion of 
VTE in this patient group if they also have COVID-19. One cannot rely on the D dimer to determine chances of VTE; you should not do that anyway even without COVID but in COVID it is likely to be much higher. If the woman is near to delivery, then the coagulation parameters and platelet count will have potential implications for delivery and guidance from a haematologist would be appropriate on an individual patient basis.

Investigation and management for suspected thrombosis should be the same as non-COVID pregnant woman.

\section{Implications for research}

Continued collection of data on specific parameters of thrombosis and haemostasis from pregnant women affected by COVID-19 is necessary to further elucidate the incidence, prognostic value, and implications of coagulopathy, and thromboembolism in pregnancy.

More detailed investigation of coagulation abnormalities may also be useful. These could include studies such as specialised factor assays (taking into account the normal haemostatic changes that occur in pregnancy).

Determination of specific cut-off values of aberrant haemostatic parameters associated with adverse outcomes in pregnancy is needed. Given the rarity of the condition, even in the face of a global pandemic, and in absence of systematic studies or until data from randomised control trials become available, international registries can be of immense value in achieving this aim. The International Society on Thrombosis and Haemostasis has developed the Pregnancy and COVID-19Associated Coagulopathy (COV-PREG-COAG) Registry, precisely to fulfil this aim. Participation in the Registry is open to health care providers worldwide and can be accessed at: https://redcap.isth.org/surveys/?s= 4JPX9W98RH.

\section{Supplementary Information}

The online version contains supplementary material available at https://doi. org/10.1186/s12884-021-03568-0.

\section{Additional file 1.}

Additional file 2: Appendix 2. D-dimer levels and platelet levels where reported for cases of COVID-19 in pregnancy.

\section{Abbreviations}

APTT: Activate partial thromboplastin time; CRP: C-reactive protein; DIC: Disseminated intravascular coagulation; DVT: Deep vein thrombosis; ICU: Intensive care unit; ISTH: International Society on Thrombosis and Haemostasis; LMWH: Low molecular weight heparin; PE: Pulmonary embolism; PT: Prothrombin time; RCOG: Royal College of Obstetrics and Gynaecology; UKOSS: UK Obstetric Surveillance System

\section{Acknowledgments}

The authors would like to thank Professor Marian Knight for her analysis of UKOSS data, for inclusion in this review.

\section{Authors' contributions}

$\mathrm{KW}$ and JT were responsible for initial study design. $\mathrm{KO}$, JT and $\mathrm{KW}$ collated reports of COVID-19 in pregnancy using methodology as per Walker et al. [15]. WL advised on overlap of cases from China. JS collected data from these sources and from cases contributed by GS, BM, SM, KM, and MO to draft the paper alongside KW. All authors were responsible for re-drafting and editing the manuscript and approved the final version.

\section{Funding}

No funding received.

Availability of data and materials

The datasets used and/or analysed during the current study available in the supplementary files.

Ethics approval and consent to participate

Not applicable.

Consent for publication

Not applicable.

\section{Competing interests}

None.

\section{Author details}

${ }^{1}$ Department of Obstetrics and Gynaecology, Nottingham University Hospitals NHS Trust, Nottingham, UK. ²Department of Haematology, Nottingham University Hospitals NHS Trust, Nottingham, UK. ${ }^{3}$ Division of Child Health, Obstetrics and Gynaecology, School of Medicine, University of Nottingham, Nottingham, UK. ${ }^{4}$ Department of Haematology, University Hospitals of Leicester, Leicester, UK. ${ }^{5}$ Division of Maternal-Fetal Medicine, Department of Obstetrics and Gynaecology, Mount Sinai Hospital, University of Toronto, Toronto, Ontario, Canada. ${ }^{6}$ Department of Biomedical and Molecular Sciences, School of Medicine, Queen's University Kingston, Kingston, Ontario, Canada. ${ }^{7}$ School of Baccalaureate Nursing, St Lawrence College, Kingston, Ontario, Canada. ${ }^{8}$ Department of Obstetrics and Gynaecology, Monash University, Clayton, Australia. ${ }^{9}$ The Irish Centre for Maternal and Child Health, University College Cork, Cork University Maternity Hospital, Cork, Ireland

Received: 16 September 2020 Accepted: 18 January 2021

Published online: 05 February 2021

\section{References}

1. Tang N, Li D, Wang X, Sun Z. Abnormal coagulation parameters are associated with poor prognosis in patients with novel coronavirus pneumonia. J Thromb Haemost. 2020;18(4):844-7.

2. Kobayashi J. Obstetrical disseminated intravascular coagulation score. Obstet Gynaecol Res. 2014;40:1500-6. https://doi.org/10.1111/jog.12426.

3. Taylor F, Toh CH, Hoots K, Wada H, Levi M. Towards definition, clinical and laboratory criteria, and a scoring system for disseminated intravascular coagulation. Thromb Haemost. 2001;86(5):1327-30.

4. Erez O, Novack L, Beer-Weisel R, et al. DIC score in pregnant women--a population based modification of the international society on thrombosis and hemostasis score. PLoS One. 2014;9(4):e93240Published 2014 Apr 11. https://doi.org/10.1371/journal.pone.009324.

5. Thachil J, Tang N, Gando S, Falanga A, Cattaneo M, Levi M, et al. ISTH interim guidance on recognition and management of coagulopathy in COVID-19. J Thromb Haemost. 2020;18(5):1023-6. https://doi.org/10. 1111/jth.14810.

6. Levi M, Thachil J, Iba T, Levy JH. Coagulation abnormalities and thrombosis in patients with COVID-19. Lancet Haematol. 2020;7(6):e438-40. https://doi. org/10.1016/S2352-3026(20)30145-9.

7. Pereira A, Cruz-Melquizo S, Adrien M, Fuentes L, Marin E, Perez-Medina T. (2020), clinical course of coronavirus Disease-2019 (COVID-19) in pregnancy. Acta Obstet Gynecol Scand. 2020;99(7):839-47. https://doi. org/10.1111/aogs.13921. 
8. Amgalan A, Othman M. Hemostatic laboratory derangements in COVID-19 with a focus on platelet count. Platelets. 2020;31(6):740-5. https://doi.org/10. 1080/09537104.2020.1768523.

9. Middeldorp S, Coppens M, van Haaps TF, Foppen M, Vlaar AP, Müller MCA et al. Incidence of venous thromboembolism in hospitalized patients with COVID-19. J Thromb Haemost. 2020Manuscript accepted online Available online 12.07.2020. https://doi.org/10.1111/jth.14888.

10. Cui S, Chen S, Li X, Shi W, Wang F. Prevalence of venous thromboembolism in patients with severe novel coronavirus pneumonia. J Thromb Haemost. 2020;18(6):1421-4. https://doi.org/10.1111/jth.14830.

11. Helms J, Tacquard C, Severac F, Leonard-Lorant I, Ohana M, Delabranche X, et al. High risk of thrombosis in patients with severe SARS-CoV-2 infection: a multicenter prospective cohort study. Intensive Care Med. 2020;46(6):108998. https://doi.org/10.1007/s00134-020-06062-x.

12. Klok FA, Kruip MJHA, van der Meer NJM, Arbous MS, Gommers DAMPJ, Kant KM, et al. Incidence of thrombotic complications in critically ill ICU patients with COVID-19. Thromb Res. 2020;191:145-7. https://doi.org/10.1016/j. thromres.2020.04.013.

13. RCOG. Coronavirus (COVID-19) infection in pregnancy. In: Information for health care professionals. London: Royal College of Obstetricians and Gynaecologists; 2020.

14. University of Birmingham. WHO collaborating Centre for Women's Health [internet] Published 28.05.2020. Accessed 06.07.2020. Available at: https:// www.birmingham.ac.uk/research/who-collaborating-centre/pregcov/about/ mother-to-child-transmission.aspx

15. Bauchner H, Golub RM, Zylke J. Editorial concern-possible reporting of the same patients with COVID-19 in different reports. JAMA. 2020;323(13):1256. https://doi.org/10.1001/jama.2020.3980.

16. Chen L, Li Q, Zheng D, Jiang H, Wei Y, Zou L. Clinical characteristics of pregnant women with Covid-19 in Wuhan. China N Engl J Med. 2020;382: e100. https://doi.org/10.1056/NEJMc2009226.

17. Walker K, O'Donoghue K, Grace N, Dorling J, Comeau J, Li W, et al. Maternal transmission of SARS-COV-2 to the neonate, and possible routes for such transmission: a systematic review and critical analysis. BJOG. 2020. https:// doi.org/10.1111/1471-0528.16362.

18. Li Y, Zhao R, Zheng S, Chen X, Wang J, Sheng $X$, et al. Lack of vertical transmission of severe acute respiratory syndrome coronavirus 2. China Emerg Infect Dis. 2020;26(6):1335-6. https://doi.org/10.3201/ eid2606.200287

19. Yu N, Li W, Kang O, Xiong Z, Wang S, Lin X, et al. Clinical features and obstetric and neonatal outcomes of pregnant patients with COVID-19 in Wuhan, China: a retrospective, single-Centre, descriptive study. Lancet Infect Dis. 2020;20(5):559-64. https://doi.org/10.1016/S14733099(20)30176-6.

20. Wang $X$, Zhou Z, Zhang J, Zhu F, Tang Y, Shen X. A case of 2019 novel coronavirus in a pregnant woman with preterm delivery. Clin Infect Dis: ciaa200. https://doi.org/10.1093/cid/ciaa200.

21. Iqbal S, Overcash R, Mokhtari N, Saeed H, Gold S, Auguste T, et al. An uncomplicated delivery in a patient with Covid-19 in the United States. N Engl J Med. 2020;382:e34. https://doi.org/10.1056/ NEJMc2007605.

22. González Romero D, Ocampo Pérez J, González Bautista L, SantanaCabrera L. Pronóstico perinatal y de la paciente embarazada con infección por COVID-19. Rev Clin EspAvailable online 4.6.2020. https:// doi.org/10.1016/j.rce.2020.04.006

23. Hong L, Smith $N$, Keerthy $M$, et al. Severe COVID-19 infection in pregnancy requiring intubation without preterm delivery: a case report. Case Rep Women's Health. 2020Available online 4.6.202. https://doi.org/ 10.1016/j.crwh.2020.e00217.

24. Blauvelt CA, Chiu C, Donovan AL, Prahl M, Shimotake TK, George RB, et al. Acute respiratory distress syndrome in a preterm pregnant patient with coronavirus disease 2019 (COVID-19). Obstet Gynecol. 2020;136(1):46-8. https://doi.org/10.1097/AOG.0000000000003949.

25. Algarroba G, Rekawek P, Vahanian SA, Khullar P, Palaia T, Peltier MR, et al. Visualisation of SARS-CoV-2 virus invading the human placenta using electron microscopy. Am J Obstet Gynecolln Press. Available 4.7.2020. https://doi.org/10.1016/j.ajog.2020.05.023.

26. Rosen $\mathrm{MH}$, Axelrad J, Hudesman D, Rubin DT, Chang S. Management of Acute Severe Ulcerative Colitis in a pregnant woman with COVID-19 infection: a case report and review of the literature. Inflamm Bowel Dis. 2020;26(7):971-3. https://doi.org/10.1093/ibd/izaa109.
27. Lokken EM, Walker CL, Delaney S, Kachikis A, Kretzer NM, Erickson A, et al. Clinical characteristics of 46 pregnant women with a SARS-CoV-2 infection in Washington state [published online ahead of print, 2020 may 18]. Am J Obstet Gynecol. 2020;S0002-9378(20)30558-5. https://doi. org/10.1016/j.ajog.2020.05.031.

28. Mehta H, Ivanovic S, Cronin A, VanBrunt L, Mistry N, Miller R, et al. Novel coronavirus related acute respiratory distress syndrome in a patient with twin pregnancy: a case report. Case Rep Women's Health. 2020:4.6.202. https://doi.org/10.1016/j.crwh.2020.e00220.

29. Mendoza M, Garcia-Ruiz I, Maiz N, Rodo C, Garcia-Manau P, Serrano B, et al. Preeclampsia-like syndrome induced by severe COVID-19: a prospective observational study. BJOGAvailable online 4.6. 2020. https:// doi.org/10.1111/1471-0528.16339.

30. Romagano MP, Guerrero K, Spillane N, Kayaalp E, Smilen SW, Alvarez M, et al. Perinatal outcomes in critically ill pregnant women with COVID-19. Am J Obstetr Gynecol MFM. 2020Available online 4.6.2020. https://doi.org/ 10.1016/j.ajogmf.2020.100151.

31. Silverstein JS, Limaye MA, Brubaker SG, Roman AS, Bautista J, Chervenak J, et al. Acute respiratory Decompensation requiring intubation in pregnant women with SARS-CoV-2 (COVID-19). AJP Rep. 2020;10(02):e169-75. https:// doi.org/10.1055/s-0040-1712925.

32. AlZaghal LA, AlZaghal N, Alomari SO, Obeidat N, Obeidat B, Hayajneh WA. Multidisciplinary team management and cesarean delivery for a Jordanian woman infected with SARS-COV-2: a case report. Case Rep Women's Health. 2020Available online 4.7.2020. https://doi.org/10.1016/j. crwh.2020.e00212.

33. Sentilhes $L$, De Marcillac F, Jouffrieau C, Kuhn P, Thuet $V$, Hansmann $Y$, et al. COVID-19 in pregnancy was associated with maternal morbidity and preterm birth. Am J Obstet Gynecol. 2020Available online 4.7.2020. https:// doi.org/10.1016/j.ajog.2020.06.022.

34. Chen S, Huang B, Luo DJ, Li X, Yang F, Zhao Y, et al. Pregnant women with new coronavirus infection: a clinical characteristics and placental pathological analysis of three cases. Zhonghua Bing Li Xue Za Zhi. 2020;49(0):E005. https://doi.org/10.3760/cma.j.cn11215120200225-00138.

35. Gidlof S, Savchenko J, Brune T, Josefsson H. COVID-19 in pregnancy with comorbidities: more liberal testing strategy is needed. Acta Obstet Gynecol Scand. 2020;99(7):948-9. https://doi.org/10.1111/aogs.13862.

36. Juusela A, Nazir M, Gimovsky M. Two cases of coronavirus 2019-related cardiomyopathy in pregnancy. AJOG MFM. 2020;2(2) supplement. https:// doi.org/10.1016/j.ajogmf.2020.100113.

37. Browne PC, Linfert JB. Perez-Jorge. Successful treatment of preterm labor in association with acute COVID-19 infection. Am J Perinatol. 2020;37(08):8668. https://doi.org/10.1055/s-0040-1709993.

38. Lu D, Sang L, Du S, Li T, Chang Y, Yang XA. Asymptomatic COVID-19 infection in late pregnancy indicated no vertical transmission. J Med Virol. 2020. https://doi.org/10.1002/jmv.25927.

39. Hirshberg A, Kern-Goldberger AR, Levine LD, Pierce-Williams R, Short W, Parry $S$, et al. Care of Critically ill pregnant patients with coronavirus disease 2019: a case series. Am J Obstet Gynecol. 2020; S0002-9378(20)30515-9. https://doi.org/10.1016/j.ajog.2020.04.029 Online ahead of print.

40. Hantoushzadeh S, Shamshirsaz AA, Aleyasin A, Seferovic MD, Aski SK, Arian SE, et al. Maternal death due to COVID-19. AJOG. 2020;223(1):109. https:// doi.org/10.1016/j.ajog.2020.04.030.

41. Rabinovich A, Abdul-Kadir R, Thachil J, Iba T, Othman M, Erez O, DIC in obstetrics: diagnostic score, highlights in management, and international registry-communication from the DIC and Women's health SSCs of the International Society of Thrombosis and Haemostasis 2019 https://doi.org/ 10.1111/jth.14523

42. Koumoutsea EV, Vivanti AJ, Shehata N, Benachi A, Le Gouez A, Desconclois C, et al. COVID-19 and acute coagulopathy in pregnancy. J Thromb Haemost. 2020;18(7):1648-52. https://doi.org/10.1111/jth.14856.

43. Goodacre S, Horspool K, Nelson-Piercy C, Knight M, Shephard N, Lecky F. Et al; DiPEP research group. The DiPEP study: an observational study of the diagnostic accuracy of clinical assessment, D-dimer and chest $\mathrm{x}$ ray for suspected pulmonary embolism in pregnancy and postpartum. BJOG. 2019;126(3):383-92. https://doi.org/10.1111/1471-0528.15286 Epub 2018 Jun 14.

44. van der Pol LM, Tromeur C, Bistervels IM, Ni Ainele F, van Bemmel T, Bertoletti $L$, et al. Pregnancy-adapted YEARS algorithm for diagnosis of 
suspected pulmonary embolism. N Engl J Med. 2019;380(12):1139-49. https://doi.org/10.1056/NEJMoa1813865.

45. Amgalan A, Allen T, Othman M, Ahmadzia HK. Systematic review of viscoelastic testing (TEG/ROTEM) in obstetrics and recommendations from the Women's SSC of the ISTH. J Thromb Haemost[epub ahead of print]. https://doi.org/10.1111/jth.14882.

46. Guan W, Ni Z, Hu Y, Liang W, Ou C, He J, et al. Clinical characteristics of coronavirus disease 2019 in China. NEJM. 2020;382:1708-20. https://doi.org/ 10.1056/NEJMoa2002032

\section{Publisher's Note}

Springer Nature remains neutral with regard to jurisdictional claims in published maps and institutional affiliations.

Ready to submit your research? Choose BMC and benefit from:

- fast, convenient online submission

- thorough peer review by experienced researchers in your field

- rapid publication on acceptance

- support for research data, including large and complex data types

- gold Open Access which fosters wider collaboration and increased citations

- maximum visibility for your research: over $100 \mathrm{M}$ website views per year

At $B M C$, research is always in progress.

Learn more biomedcentral.com/submissions 